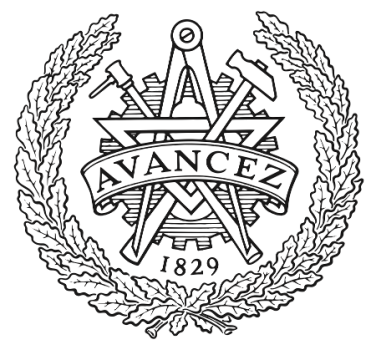

CHALMERS

UNIVERSITY OF TECHNOLOGY

\title{
Radio Localization and Mapping with Reconfigurable Intelligent Surfaces: Challenges, Opportunities, and Research Directions
}

Downloaded from: https://research.chalmers.se, 2023-04-26 14:21 UTC

Citation for the original published paper (version of record):

Wymeersch, H., He, J., Denis, B. et al (2020). Radio Localization and Mapping with Reconfigurable Intelligent Surfaces: Challenges,

Opportunities, and Research Directions. IEEE Vehicular Technology Magazine, 15(4): 52-61.

http://dx.doi.org/10.1109/MVT.2020.3023682

N.B. When citing this work, cite the original published paper.

C2020 IEEE. Personal use of this material is permitted.

However, permission to reprint/republish this material for advertising or promotional purposes 


\title{
Radio Localization and Mapping with Reconfigurable Intelligent Surfaces
}

\author{
Henk Wymeersch*, Jiguang He $^{\dagger}$, Benoît Denis ${ }^{\ddagger}$, Antonio Clemente ${ }^{\ddagger}$, Markku Juntti ${ }^{\dagger}$ \\ ${ }^{*}$ Chalmers University of Technology, ${ }^{\dagger}$ University of Oulu, ${ }^{\ddagger}$ CEA-Leti
}

\begin{abstract}
G radio at millimeter wave (mmWave) and beyond 5G concepts at 0.1-1 THz can exploit angle and delay measurements for localization, by the virtue of increased bandwidth and large antenna arrays but are limited in terms of blockage caused by obstacles. Reconfigurable intelligent surfaces (RISs) are seen as a transformative technology that can control the physical propagation environment in which they are embedded by passively reflecting radio waves in preferred directions, or actively sense this environment in receive or transmit mode. Whereas such RISs have been mainly intended for communication purposes, they can have great benefits in terms of performance, energy consumption, and cost for localization and mapping. These benefits as well as associated challenges are the main topics of this paper.
\end{abstract}

\section{INTRODUCTION}

The interaction between the digital and physical world relies on high-definition situational awareness, i.e., the ability of a device to determine its own location, as well as the location of objects and other devices in the operating environment. Applications include automated vehicles and robots in general, as well as healthcare, highly immersive virtual and augmented reality, or new human-to-machine interfaces. Situational awareness can be achieved by a variety of technologies, depending on the application and requirements, including lidars, inertial measurement units, or cameras, but also radio-based technologies, such as satellite positioning, radar, ultra wideband (UWB), cellular or WiFi. Radio-based technologies are attractive as they can have dual communication and sensing functionalities and are often less susceptible to environmental factors such as poor lighting. Since $4 G$, dedicated localization reference signals have been considered as part of communications system design and standardization.

Henk Wymeersch was supported by the Swedish Research Council under grant 2018-03701. The work by Jiguang He and Markku Juntti has been supported in part by the IIoT Connectivity for Mechanical Systems (ICONICAL) project and 6Genesis Flagship (grant 318927), both funded by the Academy of Finland, and in part by Horizon 2020, European Union's Framework Programme for Research and Innovation, under grant agreement no. 871464 (ARIADNE).

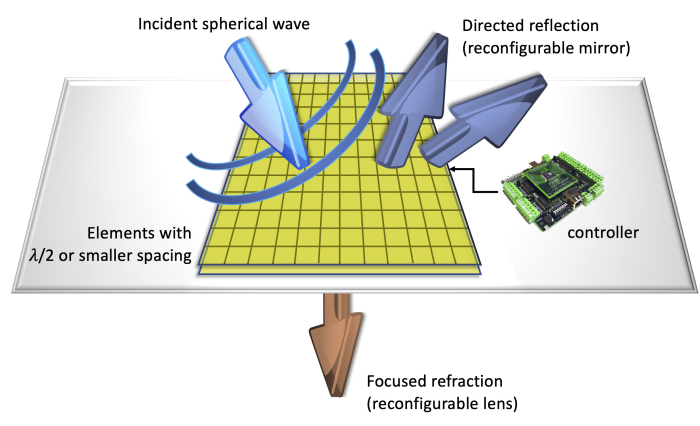

Figure 1. Example of a RIS, where a controller adjusts individual elements. Depending on the technology, the RIS can change the direction of reflections or refract the signal, similar to a lens.

These can enable location accuracy levels on the order of $10 \mathrm{~m}$. With 5G, the use of larger bandwidths and higher carrier frequencies in combination with antenna arrays at the user equipment (UE) and base station (BS) is expected to further improve the location accuracy to around $1 \mathrm{~m}$. Within Beyond 5G systems, the trend is to operate at much higher frequencies (above $30 \mathrm{GHz}$, possibly up to $1 \mathrm{THz}$ ) benefit from large available bandwidths and thus achieve even better localization accuracy. Propagation at high carrier frequencies suffers from obstructions due to objects blocking line-of-sight (LoS) path between the transmitter and the receiver. The reliance on the LoS path can be reduced through multipathaided localization by exploiting either a prior map information or through joint localization and mapping [1]. Therein, the locations of objects in the environment (surfaces and scatter points) are determined simultaneously with the user's location, a process called radio-based simultaneous localization and mapping (SLAM). Even if these solutions make use of the multipath channel as a constructive source of information in the localization problem geometry, the related electromagnetic (EM) interactions (induced by the physical environment) still remain uncontrolled and as such, largely sub-optimal from a localization perspective.

Reconfigurable intelligent surfaces (RISs) represent a break- 


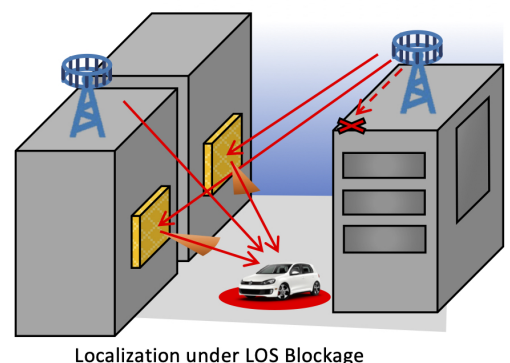

Localization under LOS Blockage

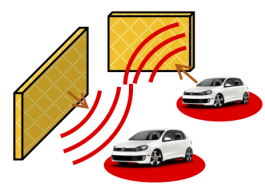

Near-field Localization

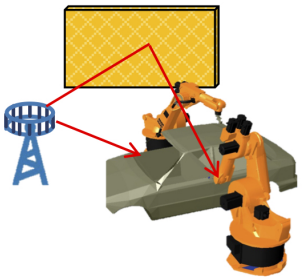

Localization in Industry 4.0

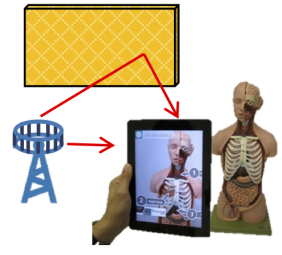

Augmented Reality

Figure 2. Application examples of RIS-based localization and mapping services (from left to right): (i) LoS blockages can be circumvented to improve localization accuracy and continuity; (ii) wavefront curvature in the near-field of a large RIS receiver or transmitter can be exploited to solve for nuisance parameters (e.g., clock biases); (iii) by creating strong and consistent multipath, RISs can support localization in very harsh indoor environment, dynamically accounting for object movements; (iv) new delay-sensitive, ultra-accurate applications will be supported by the fact that RISs do not introduce processing delays.

through technology whereby surfaces are endowed with the capability to actively modify the impinging electromagnetic wave [2], as visualized in Figure 1. A RIS can be implemented using a variety of technologies as discussed below and can provide significant benefits in terms of communication by guaranteeing coverage when the LoS is blocked. A RIS can operate as a reconfigurable mirror or as a reconfigurable lens (see Figure 1). The RIS is controlled by a local control unit that adjusts the phase profile or current distribution. Based on these fundamental operating modes, a RIS can act as a transmitter [2], receiver [3], or as an anomalous reflector, where the direction of the reflected wave is no longer specular according to natural reflection laws but steerable [4], [5]. The RIS concept can be applied at different wavelengths, ranging from low sub- $6 \mathrm{GHz}$ bands, where the technology is well understood and commercial systems are available, to $28 \mathrm{GHz}$ mmWave bands, where RISs can provide significant benefits in terms of coverage but where the technology is less mature. Finally, in the $0.1-1 \mathrm{THz}$ regime, severe path loss, higher susceptibility to blockages, atmospheric absorption, and rain attenuation as well as significant hardware limitations make RIS design challenging but can also lead to large performance gains.

The aforementioned properties and their close relation to the environment geometry make RIS attractive for localization and mapping. The potential of RIS for localization has received only limited coverage in the literature, including preliminary studies where the RIS operates in receive mode as a lens [6] and in reflection mode [7]. Hence, it is timely to delve deeper into the potential of RIS for localization and mapping, as well as the main research questions that we should address in the coming years. Possible applications of RIS for localization are visualized in Figure 2.

This paper aims to take a broader view than the technical contributions in [6], [7] by describing the core technical challenges of applying RISs to localization and mapping, along with a preliminary system vision, results, and solutions recently put forward on related topics.

\section{RADIO LOCALIZATION AND MAPPING}

\section{Basic Principles}

Any radio localization and mapping system comprises three essential parts: measurements, a reference system, and the inference algorithms.

Measurements: The measurements are derived from the radio signal between a transmitter and a receiver. They can typically be obtained directly from the channel estimation routine used for communication. Common location-dependent metrics are based on received signal strength, time of arrival (ToA), phase of arrival (PoA), angle of arrival (AoA), angle of departure $(\mathrm{AoD})$, and Doppler measurements. Measurements can be characterized by their resolution and accuracy. The resolution refers to the ability to distinguish two signals based on their measurements and depends on the signal bandwidth and duration, carrier frequency, the number of antennas, and coherent integration time. The accuracy refers to the extent to which we can determine the parameter of interest. It depends also on the signal-to-noise ratio (SNR), as well as on the detailed properties of the signal waveform such as the timefrequency and spatial power allocation.

Reference System: All the measurements are taken in a certain frame of reference, e.g., that of the receiver. References, sometimes called anchor points, have known states. There may be multiple position references, as in cellular localization or satellite positioning, which may in turn place requirements in terms of synchronization, array calibration, as well as dedicated control signals. The geometric placement of the reference plays an important role in the accuracy of a 
localization system, an effect commonly measured through the geometric dilution of precision (GDOP).

Localization and Mapping Algorithms: An important distinction between a communication and a localization algorithm is how the multipath is treated. In communication, multipath is used to provide diversity or spatial multiplexing, thus, decreasing the error rate or increasing the data rate. In localization, only the LoS has traditionally been used, as the measurements associated with that path could directly be related to the location of the user. Modern approaches also exploit measurements from non-line-of-sight (NLoS) paths, corresponding to scattered or reflected signal components [1]. A critical component in SLAM is the association of measurements to their sources, where a source can be a transmitter or a fixed object in the environment, or clutter.

In the design aspects of the measurements, reference system, and algorithms, fundamental performance bounds can play an important role. They allow us to assess the localization potential of signals or reference systems, guide the development and benchmarking of algorithms, or can even be used as approximated performance indicators or real-time optimization/selection criteria.

\section{Localization and Mapping with RIS}

The inclusion of RIS affects the three above-mentioned parts of radio localization. The measurements are in general tuples of ToA, PoA, AoD, AoA, and the Doppler shift. The relation between the measurements depends on the underlying channel model, which is largely geometric: each path corresponds to a cluster of rays, depending on the EM properties of the objects. In other words, the locations and EM properties of the environment impose a mapping from position space to measurement space. Whether this mapping is resolvable depends on the available bandwidth and number of antennas. While RIS can be used at sub- $6 \mathrm{GHz}$, the larger bandwidths at frequencies beyond $28 \mathrm{GHz}$, combined with more dense packing of RIS elements are particularly conducive to localization and mapping. The references include the BS and RIS, which can reasonably be assumed to have a pre-programmed known location and orientation in a common coordinate system, while users and passive objects have an unknown or partially known location and orientation information. The signal from the BS is to a large extent controllable in the time, frequency, and spatial domains. Therefore, it can be optimized in terms of power allocation and beamforming to maximize the accuracy of the measurements. The signals from the RIS can be shaped by the
RIS controller, to further improve accuracy, when the RIS is acting as a transmitter or a reflector [6], [7]. The design may however be less flexible than the signal from a conventional $\mathrm{BS}$, for obvious power and complexity considerations. In terms of inference algorithms, RIS-based SLAM should harness the flexibility of the BS signals and RIS controllability, to improve not only localization and mapping coverage but also accuracy.

\section{CHALLENGES AND OPPORTUNITIES}

When a RIS is used as a reflector, it could be interpreted in two different ways: as part of the passive environment, acting like any scatterer or reflector, or alternatively as part of the infrastructure, playing a similar role as a global reference or anchor point. These two views lead to fundamental challenges and opportunities (in terms of applications and research directions) in incorporating RIS in radio localization and mapping, as highlighted below. Many of these challenges are interrelated, but are presented as separate for reasons of clarity: RIS and channel modeling, near-field propagation, channel estimation, system architecture and signaling, RIS control, waveform and codebook design, and SLAM methods. While our focus is mainly on reflecting RIS, many of the opportunities and challenges are present also when the RIS is employed as a transmitter or receiver.

\section{RIS Modeling and Channel Modeling}

Challenge: There are several different antenna technologies and terminologies for RIS, including reflectarrays [8], transmit arrays [9], smart, programmable, or software-defined metasurfaces [10], large intelligent surfaces (LISs), etc. Making their usage truly ubiquitous, programmable wireless environments could be created [11]. Proper models of their functionality or how they interact with EM waves still represent an active area of research. As in the case of the beamforming, RISs could be implemented as full-digital, hybrid or analog architectures with both amplitude and phase or phase-only control. Quasicontinuous phase quantization could be selected as a function of the required complexity and power consumption specifications.

In the RIS model, the radiation pattern in azimuth and elevation should account for the coupling among the RIS elements, which are typically located on a regular or triangular lattice with an inter-element distance ranging between onetenth and one-half wavelength. Impedance matching, reflection and refraction losses can affect RIS performance. In the case of reflect and transmit arrays, for example, the scattering 
properties of the elements should be included in the model. The impact of the oblique incidence on the element performance is also an important parameter. More generally, this RIS model should be defined according to the EM properties of the chosen underlying technology (e.g., specific EM synthesis tools are needed to calculate the impedance modulation in case of metasurfaces). RIS geometry and periodicity, which impact the mutual coupling between its constituting elements, should also be taken into account. Finally, the method and electronics used to control the RIS beam (e.g., single frequency phase-shift, time delays, quasi-continuous phase vs. quantized phase) should be properly developed, while considering related hardware impairments (e.g., specific models for phase-shifters and other building tunable devices, including RF losses and limited resolution, active element performances). The model of the radio channel to and from a RIS, including the beam shape of signals, polarization effects, path loss, as well as joint angular and delay spread and how to control these require significant research efforts. Moreover, the interaction with new BS technologies and radio stripes is poorly understood. Hardware impairments will be more pronounced the higher the carrier frequency, which in turn impacts the amount of flexibility and control needed.

While a more in-depth overview of different technologies can be found in the literature for reflectarrays [8], transmitarrays [12], and phased arrays [13], a specific example of RIS based on transmitarray technology is presented in Figure 3 . This antenna is composed of a controllable flat lens with $20 \times 20$ elements, having an aperture size of $102 \times 102 \mathrm{~mm}^{2}$ and a spatial feed based on a 16-element substrate integrated waveguide (SIW) array [9], located at a distance of $30 \mathrm{~mm}$ from the flat lens aperture. A beam can be electronically controlled with 1-bit phase quantization at a commutation speed in the range of $5-10 \mathrm{~ms}$. The aperture efficiency corresponds to a realized gain between $20-23 \mathrm{dBi}$, which can be improved up to $40 \%$ with 2-bit designs.

Opportunities: Determining proper models requires a combination of skills, ranging from the EM theory to circuits. Since there are multiple RIS technologies and a RIS can act in transmit, receive, or reflect mode, there is no one-size-fitsall model. What is common in all these models, however, is the dependence on location, orientation, and extent of the RIS, leading to clear opportunities to use the models for localization purposes, where each specific model of RIS may present different opportunities to improve localization and mapping. In addition, if models are to be used for localization and mapping,
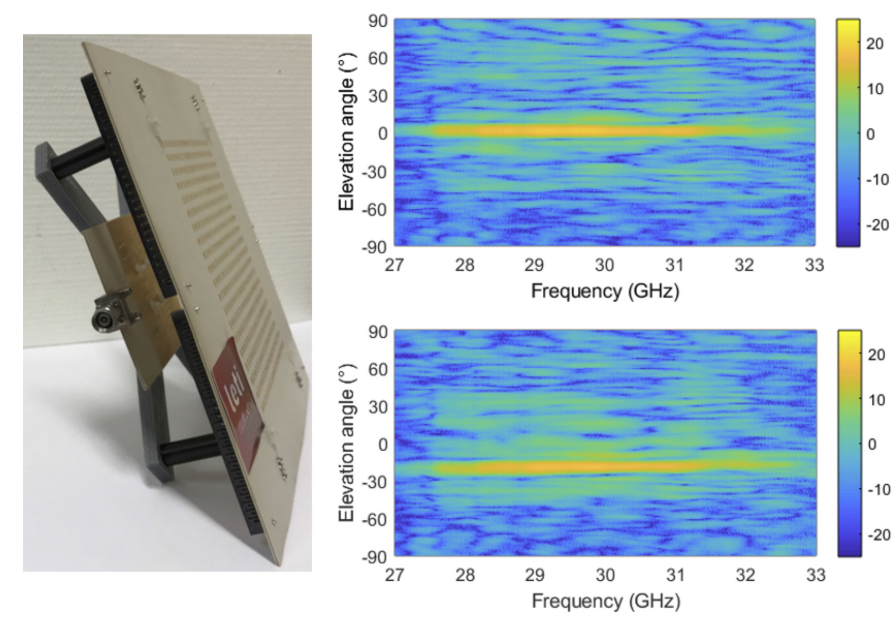

Figure 3. Ka-band RIS based on electronically steerable transmitarray architecture. (Left) $20 \times 20$-element RIS with 800 p-i-n diodes and a substrate integrated wave-guide (SIW) spatial feed [9]. (Right) Measured copolarization beams (gain in $\mathrm{dBi}$ ) on the $0^{\circ}$-azimuth cut-plane at broadside direction and scan angle of $-20^{\circ}$ as a function of the elevation angle and frequency.

they should be spatially and temporally consistent and account for the locations and orientations of all relevant objects (both passive and active). Models are likely to differ, depending on the frequency band, but multi-band operation can benefit from models that are consistent across a very wide frequency range.

\section{Near-field Propagation}

Challenge: Beyond the Fraunhofer distance, signals are in far-field so that the plane wave assumption holds. The nearfield region is proportional to the surface area of the RIS, so that a $20 \mathrm{~cm} \times 20 \mathrm{~cm}$ RIS has an 8 meter near-field region at a wavelength of $1 \mathrm{~cm}$. Hence, even at moderate distances to the RIS, near-field propagation occurs, leading to wavefront curvature, which must be properly modeled and accounted for in the communication system. This affects both RIS and channel modeling as well as RIS channel estimation and control.

Opportunities: The wavefront curvature (see also Figure 2) can be harnessed to reduce the need for infrastructure or synchronization. The PoA from a near-field signal provides information about both the angle and distance to the RIS so that in combination with ToA it is possible to determine unknown clock biases. The PoA observable by an array of elements, possibly asynchronous and non-coherent to the transmitter itself, can also be exploited directly in terms of spherical wave localization. This exploitation requires novel dedicated signal processing methods, as well as possibly new signal designs that can maximally harness the near-field properties. The specific properties of different RISs (e.g., their size) can be 
used in near-field multipath-aided positioning and to simplify data association in SLAM.

\section{Channel Estimation}

Challenge: In communication, for the purpose of detection, phase adjustment, or precoding, RIS channel estimation is needed in receive, reflect, or transmit mode, respectively. For localization, the compound channel needs to be estimated at the receiver side, in order to extract the AoA, AoD, and ToA of each propagation path (or cluster), as well as their respective spreads. As a RIS may have limited processing capabilities and, under reflect mode, may have no or few RF chains, such channel estimation to and from the RIS is challenging [3]. For instance, in [14], a protocol is proposed to separately estimate the LoS and RIS channels, by activating the RIS with different phase patterns while sending pilots leading to delays. Channel estimation in receive mode is arguably not well understood, with, e.g., [15] analyzing the impact of channel estimation errors, but not proposing a channel estimation routine.

Opportunities: At high carrier frequencies, the channel response is sparse and depends mainly on the geometric configuration of UE, BS, and the environment (including the RIS). Hence, the sparse channel properties can be leveraged in the process of channel parameter estimation by resorting to compressive sensing (CS) methods [3]. The estimated channel parameters in turn help to determine the user location via the 3D geometrical relationships. Prior location information of the UE and the RIS location and orientation could be used as a proxy for channel state information (CSI). In other words, the geometric information could be converted to partial CSI or to CSI statistics. For instance, the end-to-end compound channel can be determined a priori as a function of the UE location through machine learning techniques. As the UE location is generally only statistically known, this uncertainty should be reflected in the CSI uncertainty accordingly. Hence, suitable Bayesian methods are needed to provide this mapping.

\section{Signaling and System Architecture}

Challenge: Localization can be performed in uplink, downlink, or sidelink (i.e., between two UEs). Uplink localization can benefit from richer measurements and more processing power at the BS side, while downlink localization can reuse high-power downlink pilots, localize multiple users simultaneously, and requires less UE power. Sidelink signals can be used for relative localization, both in a bistatic and a monostatic configuration. No matter which architecture is chosen, control and feedback signals need to be provided among all network entities. Calibration and synchronization signals are needed for maintaining coherence among the position references. This can be performed over the air or via wired links between the infrastructure elements. Finally, fine a priori location and orientation information of RIS is needed to support localization. For static RIS, this can be achieved by a one-time surveying step or by the use of GPS signals when available. For mobile RIS, the architecture should support RIS tracking methods. Tracking multiple users with multiple RIS requires additional inter-RIS coordination and control signaling.

Opportunities: The design of signaling protocols and the trade-offs of uplink, downlink, and sidelink RIS-aided localization are still unknown and remain largely unexplored in the research community. A possible architecture with the corresponding signal flow is depicted in Figure 4. The estimated UE location information can be re-injected to refine the RIS setting and selection to further improve the next localization steps. As RISs are expected to often operate with obstructed LoS, localization and mapping methods can support communication by providing the system with a prediction of the future LoS conditions. While physical RIS placement will be limited by the environment and legal restrictions, RISs can still be downselected, activated, and optimized jointly for communication and localization performance.

\section{RIS Control}

Challenge: RIS control refers to adjusting the surface impedances to steer the beams. Efficient RIS control depends on the connection to other network elements and related communication latency constraints. The material and hardware properties will set practical limits to the accuracy and speed of the phase shift control, which is in practice often quantized to finite accuracy. This may easily lead to combinatorial optimization problems. The control mechanisms and material properties have an impact on the RIS power consumption and thereby the overall system energy efficiency. All this raises research questions on how frequent the control can and should be updated (e.g., frame-level or symbol-level). This is further compounded by the possible mobility of RIS, which requires dedicated tracking routines. The RIS can operate as a transmitter, a receiver, or a reflector, where each mode poses its own control challenges. For instance, a RIS lens must control both RIS phases and switches for optimal performance.

Opportunities: In contrast to communication, localization and mapping applications can be supported with low update 


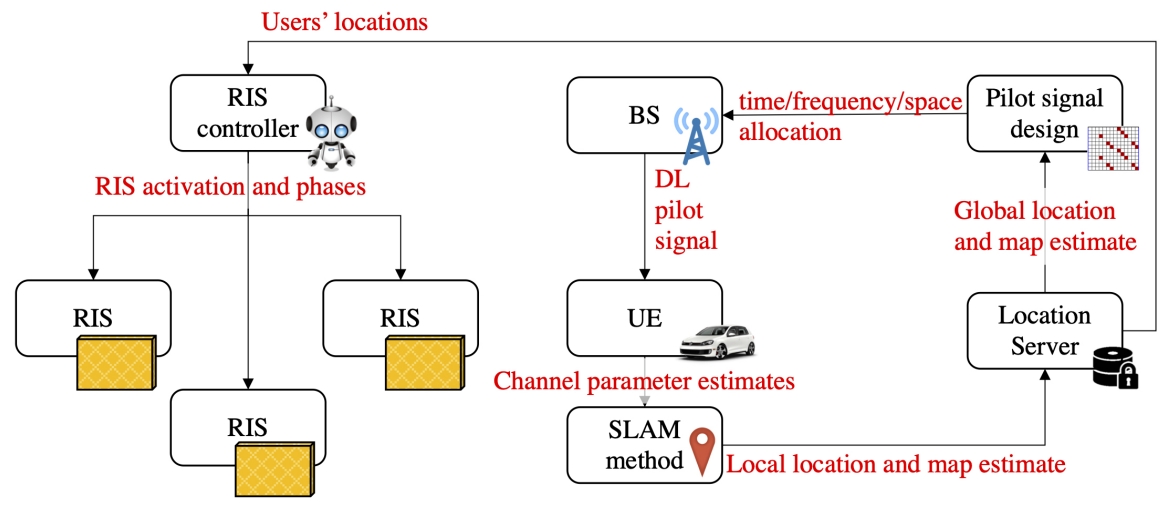

Figure 4. Possible flowchart of signaling for downlink RIS-aided localization and mapping. A priori user location information is used to determine which RIS to activate and how to set its phases. The downlink (DL) pilot signal, reflected by the RIS, is optimized given the current UE and environment conditions, and is used by the UE to estimate the channel parameters. These are fed to the SLAM algorithm, which determines the UE location and local map. Maps from different UEs can be fused to provide global situational awareness.

rates, related to physical movements of the UE and environment, and hence infrequent RIS control. Each RIS with known a priori location provides an additional source of information, though RIS signals should consider multipath resolvability to avoid harmful self-interference of the controlled multipath components from the RIS. A priori map information, in combination with the UE location, can be leveraged to decide which RIS to activate and control, while forcing other RIS to direct signals away from the UE. Allowing limited feedback from the UE or BS helps the RIS design, e.g., phase and/or amplitude, based on predetermined codebooks at the RIS [7]. The control decisions for communication will be different from those for localization, since for communication the SNR and data rate are the main metrics, while for localization accuracy and continuity are the most important. To this end, RISs can be controlled to optimize the GDOP or other localization-relevant metrics. RIS control also allows reflecting an incoming signal towards multiple directions simultaneously, providing multiuser localization support from a single base station, as well independent reflections using different polarizations, frequency band, or sub-array architectures. Finally, the RIS activation schedule can be a tool to dim or illuminate (and thus map) parts of the environment that are not accessible by the BS.

\section{Waveform and RIS Codebook Design}

Challenge: The CSI or its proxy via location information needs to be used for the design of beamforming at the BS, RIS, and UE. As in standard mmWave communication, the design should be robust to account for location estimation errors, which include both position and orientation. In addition, finite quantization of the RIS phases, which enables low-power lowcomplexity control as mentioned above, adversely limits the flexibility of the codebooks that can be used. The waveform design at the BS should also account for the presence of the RIS and availability of the LoS path [5], [14].

Opportunities: Similar to standard position reference signals in LTE and 5G, dedicated waveforms can be designed for localization with or without RIS. Such joint designs involve both waveforms at the BS as well as the codebooks at the RIS and should be sufficiently flexible to support accurate angle or delay estimation. The uncertainty in the map and UE location can be accounted for through robust designs, which may explicitly encode different levels of location uncertainty. For a RIS transmitter, waveform design remains an unexplored area, while for a RIS reflector, preliminary results [7] indicate the potential of dedicated designs. In particular, for the nearfield, the RIS codebook can be configured to direct (or receive) power to (from) a certain location, rather than to a certain direction in far-field. Figure 5 shows the performance of different codebooks at the UE and RIS, where a hierarchical codebook brings promising performance in terms of mean squared error (MSE) with low training overhead and approaches that of the exhaustive search with the highest resolution codebook even in the low SNR regime.

\section{Localization and Mapping Algorithms}

Challenge: Recovery of the user's position and the map of the environment is based on the multipath signal information. As signal paths parameterized by their angles and delays have no identifier of the corresponding source, this process also involves a data association of the detected paths to RIS as well as passive objects in the environment. In the presence of clutter measurements and missed detections due to directional beamforming, this is not an easy task. As the state of the user 


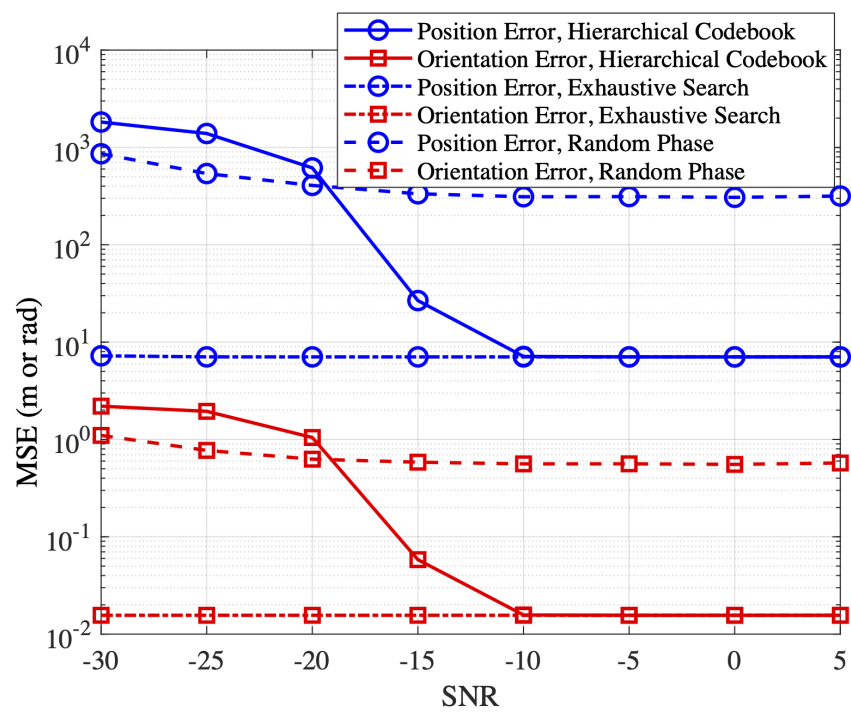

Figure 5. Comparison on localization performance in terms of position error and orientation error with codebook-based schemes [7]: hierarchical codebook at both UE and RIS, exhaustive search with highest-resolution codebooks at both UE and RIS, Random phase at RIS and highest-resolution codebook at UE. Parameters: BS located at [0, 0], RIS located at [40,60], and MS located at $[60,45]$. The number of antennas at BS and MS are 64 and 16, respectively, while the RIS has 16 units. The orientation of UE is $\pi / 10$ and the LoS path between BS and UE is blocked.

contains both the 3D position and 3D orientation, as well as a clock bias, a sufficient number of resolvable signal paths must be available, which must be enabled by proper RIS control. When the user and possibly also the RIS is mobile, dedicated tracking routines that include mobility models must be applied. For RIS receivers, separating different users and objects, as well as associating landmarks across different RIS receivers will be computationally challenging.

Opportunities: The use of RIS has clear benefits for localization and mapping algorithms since their location and orientation are known a priori. While data association is still needed to separate RIS signal paths from non-RIS signal paths, a priori information reduces the number of data association hypotheses and allows better localization of passive landmarks and users. These benefits are present in monostatic as well as bistatic configurations, not only in terms of localization accuracy but also in service coverage. As for algorithm design, various solutions have been put forward for multipath-aided localization or channel-SLAM. Those could be extended to the multi-RIS context. Among such algorithmic proposals, the solutions based on Bayesian inference over factor graphs and message-passing techniques look particularly suitable and promising, given the complexity of the new RIS-based SLAM problem (i.e., with the necessity to revolve and process signal contributions from multiple heterogeneous sources, possibly within strongly asymmetric and/or cooperative system set- tings) [1]. Finally, proper algorithm design should include all aforementioned challenges in RIS localization and mapping to reap the full potential of the RIS.

\section{Comparison of RIS and Passive Objects}

To conclude this section, we compare in Figure 6 the theoretical error bounds for ToA-based localization over a canonical scene as a function of the actual UE location in five distinct scenarios: one BS and one "natural" scatter point, one BS and one passive reflecting surface, two BSs (each with $50 \%$ of the bandwidth), 1 RIS with a scatter-like model per element (Model 1), and 1 RIS with a reflector-like model per element (Model 2). Both RIS models are considered in the far-field regime, for simplicity. Despite the use of a single RIS in our example, it is shown that the RIS exhibiting a behavior according to Model 1 already provides limited - yet interesting - gains in terms of both coverage, compared to a passive reflector, and localization error, when compared to a passive scatterer. The use of a more advanced RIS according to Model 2 could even lead to much better performance in terms of both coverage and errors, comparable to 2 active BSs. Another major expected benefit from using RISs instead of passive objects (not illustrated herein for brevity), lies in the capability to ensure service continuity and service-adaptive localization quality, even in case of a blocked LoS link.

\section{CONCLUSIONS AND OUTLOOK}

We have argued that RISs can be beneficial for localization and mapping in terms of improved accuracy or extended physical coverage, provided the appropriate models and algorithms can be developed. Progress in this area is somewhat hampered by the immaturity of working assumptions and models, which would need further investigation and validations. Different visions of the RIS coexist today, depending on their technological maturity, leading to distinct physical behaviors (typically, in terms of end-to-end power loss over reflected paths), and, thus, distinct advantages and drawbacks with respect to localization and mapping. Beyond this, the actual feasibility of integrating and controlling the RIS at a low monetary cost, low power, low complexity, and low overhead, and, possibly, the necessity to acquire side channels or prior UE location for optimal control, are still challenged by more conventional approaches such as deploying additional BSs or relays.

The overall aim of this paper was to provide the reader with an up-to-date overview of RIS-based localization and 

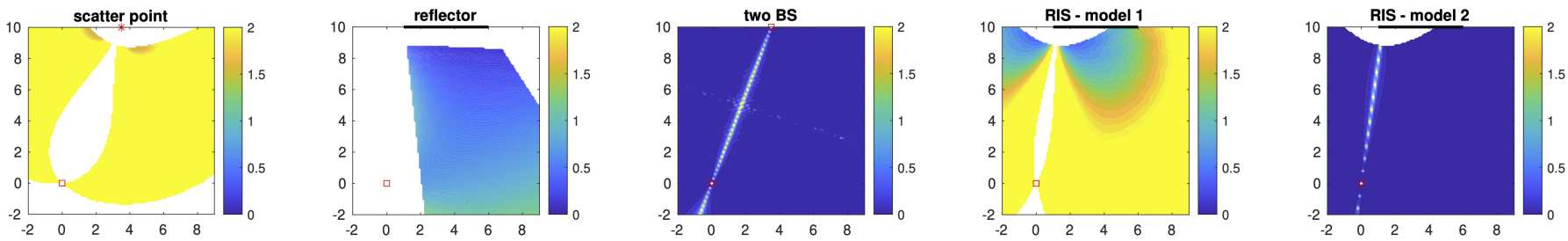

Figure 6. Comparison of far-field localization performance in terms of position error bound (shown in colors, with numbers expressed in meters) for 5 scenarios: $1 \mathrm{BS}+1$ scatter point, $1 \mathrm{BS}+1$ passive reflecting surface, 2 BS (each with $50 \%$ of the bandwidth), 1 RIS with a scatter-like path loss model per element (model 1), and 1 RIS with a reflector-like path loss model per element (model 2). Parameters: BS located as [0,0], $28 \mathrm{GHz}$ carrier, $200 \mathrm{MHz}$ bandwidth, $1 \mathrm{~mW}$ transmit power, RIS located at $[1.3,10]$ with a phased-array structure comprising 100 elements spaced by $\lambda / 2$, scatter point with RCS of $0.01 \mathrm{~m}^{2}$, reflecting surface with $30 \%$ reflectivity.

mapping. We have also described the main challenges in this field. Moreover, we provide a large number of prominent research questions, along with potential avenues of research to answer these questions. As we usher in the era of beyond 5G communications, we believe it is time to also consider beyond $5 \mathrm{G}$ or $6 \mathrm{G}$ localization. The RIS concept can be a game-changer for next-generation localization and mapping applications and deserves attention from the communication, signal processing, propagation, and antenna communities.

\section{REFERENCES}

[1] E. Leitinger, F. Meyer, F. Hlawatsch, K. Witrisal, F. Tufvesson, and M. Z. Win, "A belief propagation algorithm for multipath-based SLAM," IEEE Trans. Wireless Commun., vol. 18, pp. 5613-5629, Dec 2019.

[2] E. Basar, M. Di Renzo, J. De Rosny, M. Debbah, M. Alouini, and R. Zhang, "Wireless communications through reconfigurable intelligent surfaces," IEEE Access, vol. 7, pp. 116753-116773, 2019.

[3] A. Taha, M. Alrabeiah, and A. Alkhateeb, "Enabling large intelligent surfaces with compressive sensing and deep learning," arXiv preprint arXiv:1904.10136, 2019.

[4] E. Björnson, O. Özdogan, and E. G. Larsson, "Intelligent reflecting surface versus decode-and-forward: How large surfaces are needed to beat relaying?," IEEE Wireless Communications Letters, vol. 9, no. 2, pp. 244-248, 2020.

[5] Q. Wu and R. Zhang, "Intelligent reflecting surface enhanced wireless network via joint active and passive beamforming," IEEE Trans. Wireless Commun., vol. 18, pp. 5394-5409, Nov 2019.

[6] S. Hu, F. Rusek, and O. Edfors, "Beyond Massive MIMO: The Potential of Positioning With Large Intelligent Surfaces," IEEE Trans. Signal Process., vol. 66, pp. 1761-1774, Apr. 2018.

[7] J. He, H. Wymeersch, T. Sanguanpuak, O. Silvén, and M. Juntti, "Adaptive beamforming design for mmwave RIS-aided joint localization and communication," in IEEE Wireless Communications and Networking Conference Workshops (WCNCW), 2020.

[8] S. V. Hum and J. Perruisseau-Carrier, "Reconfigurable reflectarrays and array lenses for dynamic antenna beam control: A review," IEEE Trans. Antennas Propag., vol. 62, pp. 183-198, Jan 2014.

[9] L. Di Palma, A. Clemente, L. Dussopt, R. Sauleau, P. Potier, and P. Pouliguen, "Circularly-polarized reconfigurable transmitarray in Kaband with beam scanning and polarization switching capabilities," IEEE Trans. Antennas Propag., vol. 65, pp. 529-540, Feb 2017.

[10] M. Bodehou, E. Martini, S. Maci, I. Huynen, and C. Craeye, "Multibeam and beam scanning with modulated metasurfaces," IEEE Trans. Antennas Propag., vol. 68, no. 3, pp. 1273-1281, 2020.
[11] C. Liaskos, A. Tsioliaridou, A. Pitsillides, S. Ioannidis, and I. F. Akyildiz, "Using any surface to realize a new paradigm for wireless communications," Communications of the ACM, vol. 61, pp. 30-33, 2018.

[12] J. R. Reis, M. Vala, and R. F. S. Caldeirinha, "Review paper on transmitarray antennas," IEEE Access, vol. 7, pp. 94171-94188, 2019.

[13] B. Sadhu, X. Gu, and A. Valdes-Garcia, "The more (antennas), the merrier: A survey of silicon-based mm-wave phased arrays using multiic scaling," IEEE Microwave Magazine, vol. 20, pp. 32-50, Dec 2019.

[14] B. Zheng and R. Zhang, "Intelligent reflecting surface-enhanced OFDM: Channel estimation and reflection optimization," IEEE Wireless Communications Letters, vol. 9, no. 4, pp. 518-522, 2020.

[15] M. Jung, W. Saad, Y. Jang, G. Kong, and S. Choi, "Performance analysis of large intelligent surfaces (LISs): Asymptotic data rate and channel hardening effects," IEEE Transactions on Wireless Communications, vol. 19, no. 3, pp. 2052-2065, 2020.

Henk Wymeersch (S'02-M'06-SM'19) received the Ph.D. degree in Electrical Engineering/Applied Sciences in 2005 from Ghent University, Belgium. $\mathrm{He}$ is currently a Professor in Communication Systems with the Department of Electrical Engineering at Chalmers University of Technology, Sweden. $\mathrm{He}$ is also a Distinguished Research Associate with Eindhoven University of Technology. Prior to joining Chalmers, he was a Postdoctoral Associate during 2006-2009 with the Laboratory for Information and Decision Systems (LIDS) at the Massachusetts Institute of Technology (MIT). He has served as Associate Editor for several IEEE Transactions.

Jiguang He received the B.Eng. degree from Harbin Institute of Technology, Harbin, China, in 2010, M.Sc. degree from Xiamen University, Xiamen, China, in 2013, and Ph.D. degree from University of Oulu, Finland, in 2018, all in communications engineering. From September 2013 to March 2015, he was with Key Laboratory of Millimeter Waves at City University of Hong Kong, working on beam tracking over millimeter wave MIMO systems. Since June 2015, he has been with Centre for Wireless Communications (CWC), University of Oulu, Finland. His research interests span millimeter wave MIMO communications, reconfigurable intelligent surfaces for joint communication and positioning. 
Benoît Denis received the E.E. (2002), M.Sc. (2002), and Ph.D. (2005) degrees in electronics and communication systems from INSA, Rennes, France. Since 2005, he has been with CEA-Leti, Grenoble, France, contributing to collaborative research projects in various domains such as wireless sensor and wearable networks, heterogeneous and cooperative networks, Ultra Wideband and (Beyond) 5G technologies, as well as mobile applications related to the Internet of Things or Intelligent Transportation Systems. His main research interests concern localization-enabled communication networks, ranging/positioning/tracking and hybrid data fusion algorithms, radio channel modeling and cross-layer protocol design.

Antonio Clemente received the Ph.D. degree in signal processing and telecommunications from the University of Rennes 1, Rennes, France, in 2012 at CEA-LETI, Grenoble, France. Since 2013, he is a Research Engineer at CEA-LETI, Grenoble, France. His current research interests include fixedbeam and electronically reconfigurable transmitarray antennas, millimeterwave and sub- $\mathrm{THz}$ antennas, antenna arrays, near-field focused systems, antenna modelling, miniature integrated antennas, antenna fundamental limitations, near-field and far-field antenna measurements. He has authored or coauthored more than 105 papers in international journals and conferences, and received 12 patents. From 2013 to 2016, he has been the technical coordinator of the H2020 joint Europe and South Korea 5GCHAMPION project.

Markku Juntti (S'93-M'98-SM'04-F'20) received his Dr.Sc. (EE) degree from University of Oulu, Oulu, Finland in 1997. In the academic year 1994-95, he was a Visiting Scholar at Rice University, Houston, Texas. In 1999-2000, he was a Senior Specialist with Nokia Networks. Dr. Juntti has been a professor of communications engineering since 2000 at University of Oulu, Centre for Wireless Communications (CWC), where he also serves as Head of CWC - Radio Technologies Research Unit. His research interests include signal processing for wireless networks as well as communication and information theory. Dr. Juntti is also an Adjunct Professor at Rice University. $\mathrm{He}$ is an Editor of IEEE TRANSACTIONS ON COMMUNICATIONS. 